


\section{Gum Geleit!}

Die Brumbriffe mollen in bas Gebiet ber Surisprubeng ein=

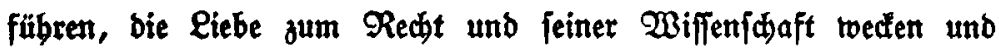
förbern, immer tieferez (Eindringen ermögliden, bem Slnterticht und bem Redtşleben bienen. Seber Grundrí bietet bie gefdlofiene $\mathfrak{D a r}$. ftellung eines Redtäftoffes und ift ein felbftändiges Rebrbud mittleren $\mathfrak{U}$ Imfangę.

Daneben joll unjer Unternebmen aut) Den Dozenten unter $=$ fiüsen, indem es ibn und ben Bortrag entlaftet. Dem Redtz= lebrer ftellt bie beutige Gpezialifierung ber Wiffenfadaft vielfac un= crmeplidise 2lufgaben. Er vermag entweder nur einen Überbliť uber ein gropes Gebiet $z^{\mathfrak{u}}$ geben - Dann feglt bie Tiefel Ober ex muß getwiffe Seile jeines Gtofftreijez weglaffen - Dann leibet bie Bolffändigleit des Gyftems! Die Grundriffe gewäbren nun bem Bortragenben bie Röglidhfeit, auf fie zu vertweijen, um anbere Teile feines Gebietes befo tiefer, eindringliaer und bamit eindruds: voller berausarbeiten zu tönnen. Go find fie berufen, eine Litufe im Slnterridt augaufülen. Sn biefem Ginne ftellen fie $\mathfrak{E} r=$ $\mathfrak{g} \mathfrak{a} \mathfrak{z} \mathfrak{u n g} \$ \mathfrak{b} \mathfrak{u} d e r d \mathfrak{d}$.

Go mögen unjere Werfe bagu beitragen, bie neue Generation

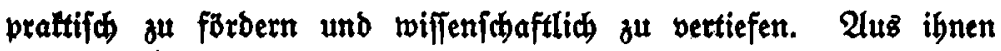

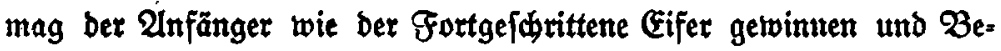
geifterung i申ßßpfen. Denn obme Begeifterung iff febes Gtubium tot.

Die Buberiidt auf Deutidlanbs funge Suriftentwelt bat fict fđon erfreulid exfült.

Die Jetausgeber. 


\section{Aug Der Sammlung

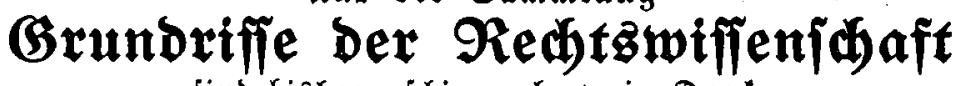

find bisher exjdienen bezw. im Drat:

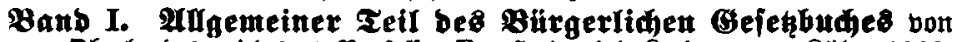

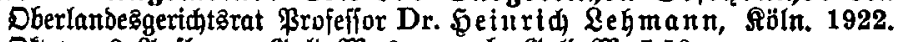
Dltab. 2. 9uflage. Bold = $\mathfrak{D}$. 6.-, geb. Bold $=\mathfrak{D C} .7 .50$.

Banb II. Enuloreht bes Burgerliqen Gefebuthes non Dber-

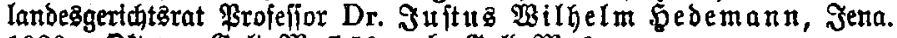

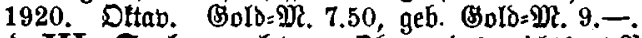

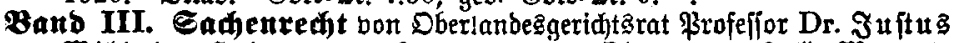
Wilgelum Sebemann, Jena. 1924. Stab. ca. Bolb= Mi. 8.50, geb. ca. Bolb= $=\mathfrak{M}$. 10.-.

Band V. Crbredht bon Geheimrat Frofeffor Dr. Frieoria Ende=

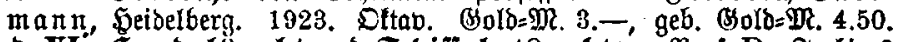

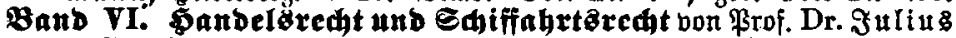

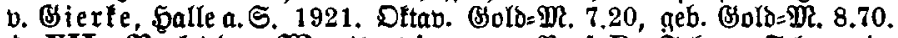

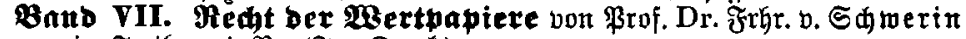
in Freiberg i. $\mathfrak{B}$. ( $(\Im \mathfrak{m}$ Druft.)

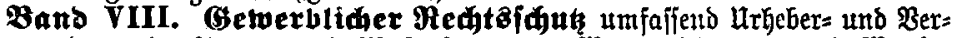

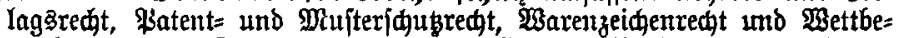
werbirecht bon Dr. jur. Ul lexan ber Elfter, Berlin. 1921. Dttav. Gold = Di. 5.-, geb. Gold = 2 . 6.50.

Qans IX. Cinfubrung in Die Pedtswiffenfaft bon Dberlanbes:

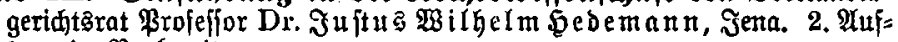
lage in Borbereitung.

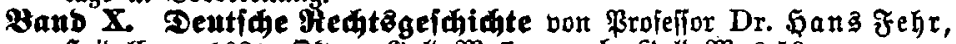

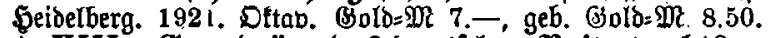

Band XIII. (Srunbzige bes beutiden \$ribatrecht8 von Profeffor

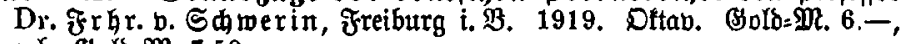
geb. Bold $=\mathbb{M}$. 7.50 .

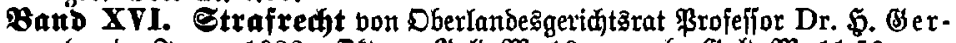

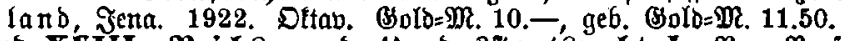

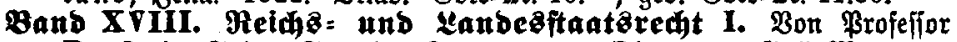
Dr. Frib Stier=Somlo, Röln. 1924. Dltab. ca. Bold=M. 12.-, geb. ca. (Gold= $\mathbb{D}$. 13.50 .

Band XIX. Reid8= und Randesftaat8redt II. Bon Brofefjor Dr. Fris Stier=Somlo. (Im Drud.)

Su übrigen find in Borbereitung:

Banb 1V. Eamiltenredt bon Dberlandes. | Bnnb XVII. Etrafprosef von Geheintat

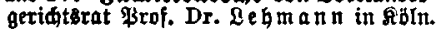

Bano XI. Grundä̈ge Det xōmtfoen Sedtegeififite.

Dand XII. Brandäge Des römilacu

Bribatredts bon Frof. Dr. Friebrid Enbemann it Geibelberg.

Panb XIV. Bfollurosefredt von \$rof. Dr. Bebmana in foln a. vih.

Banb XV. Roufures una Fuiedtungse redt bon \$rof. Dr. LeGmann in goln a. æh.

Brof. Dr. to. Bgeli $\mathrm{g}$ itl Mintinten

Banb XX u. XXI. Berwaltungsredt in zmei Zetten bon \$rof. Dr. Stier. So mio in fidn a. $\Re \mathrm{h}$.

Band XXII. Gostales Berfidexungs. redt oon Brof. Dr. Gtier. GomIo.

gandXXIII. Sogitales Grbettse u. Sand: redt. Bon Brof. Dr. Stier.Somlo. Band XXIV. Atrdearedt. Bon \$rof. Dr. G. Э. Ebers.

Mand $\mathbf{X} \bar{v}$. Bifierredt. Bon ßrof. Dr. Бerbart $\mathfrak{x}$ זaนs.

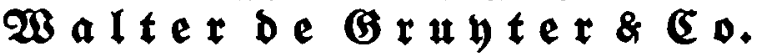 Berlin W. 10 und Reipzig}




\section{2luछ ber}

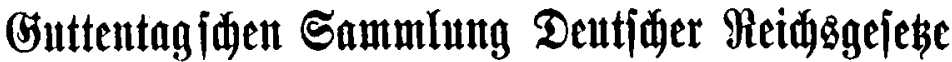

(Bolftänbiges Berzeinnis ber Gammlung toftenlos)

\section{Band 38/39:

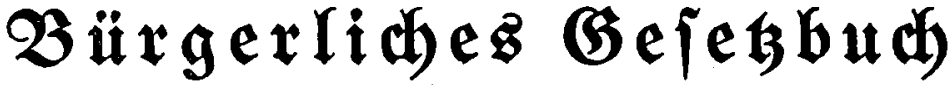 nebjt Einfübrangsgejeb}

Bon Dr. A. Uळilles. In Berbinb.mit Drof.Dr. An bré,

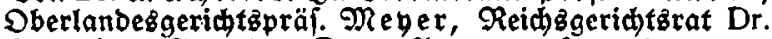
Gtreder, Gtaatsrat Dr. v. Unzzer. Serauszeg. von Birłl. Beh. Oberjultizrat u. Oberlanbezgerichtžprắtbenten M. Breiff. Elfte, vermehrte u. verbelī. 2luft. 1923. Mit

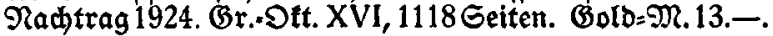

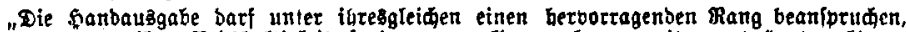

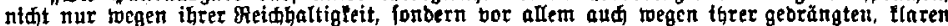
überfigten uno Borbemerlungen, in benen bie Snundüge ber Meditglebre in menigen turgen

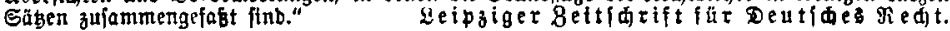

Band 11:

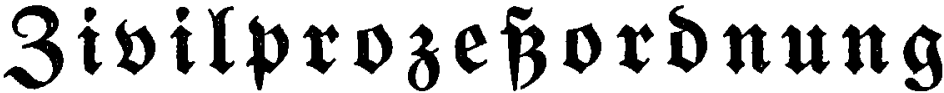

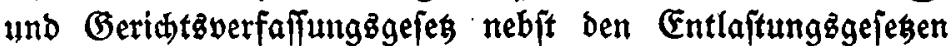
Mit Inmerturgen unter befonderer Berüffidtigung

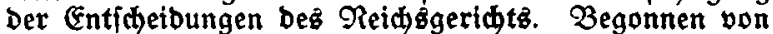

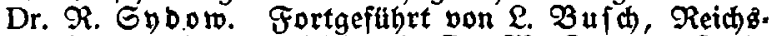

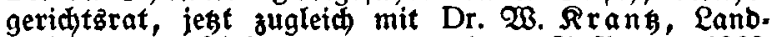
gerichtzrat.' Eiebzehnte, vermehrte 2luflage. 1922. Durb Naditrag ergänzt nad Dem Gtand yon 1924 . Groß̉. Oltab. XVI, 1178 Geiten. $\mathfrak{G o l b}=\mathfrak{R} .15$. .

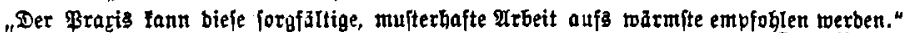
Das $\pi$ e d t.

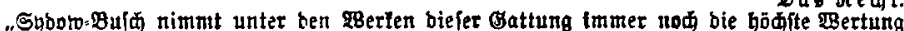

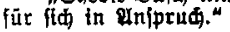

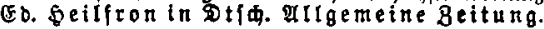

Band 4:

\section{$\mathfrak{S} \mathfrak{a} \mathfrak{n} \mathfrak{d} \mathfrak{l} \mathfrak{i} \mathfrak{g} \mathfrak{e} \mathfrak{j} \mathfrak{e} \mathfrak{z} \mathfrak{b} \mathfrak{u}$ d}

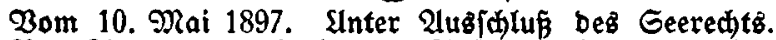
Bon Qitthauer. Gedizehnte Huflage. Seraügegeben von Prof. Dr. $\mathfrak{U}$. Mlofie, Geheimer $\mathfrak{T u j t i z r a t . ~ S I n v e r = ~}$ änberter Neubrud. 1920. Ottab. 603 Geiten. Golo-M. 6.50.

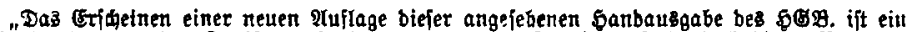

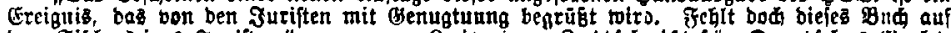
bem '

Qeipgiger Beft出ift für DeutfGes Hedt.

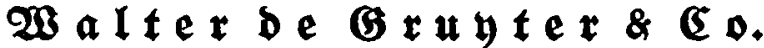
Berlin W. 10 unb Leipzig 


\section{Grundrifje \\ Der

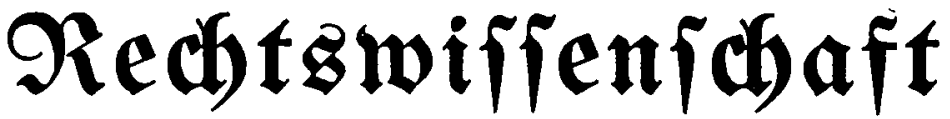

Inter Mitarbett bon

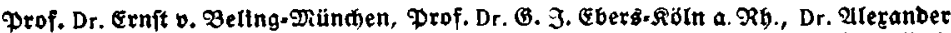
Elfter-Berlin, Prof. Dr. Frtebrth Enbemann.Setbelberg, Prof. Dr, Sanz Gebr-Selbels berg, Prof. Dr. Selnria Gerland= Jena, Prof. Dr. Gultus v. Gtertes Salle a. S., Prof.

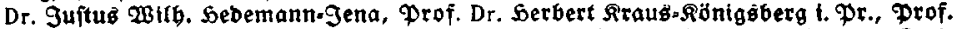
Dr. Seinrţ Lebmann = Röin a. Rb., Prof. Dr. Claubtus Greiberr v. Edwerin. Grets burg t. 23., Prof. Dr. Frth Gtter=Gomloz:ö̈n a. Ry.

beraugigegeben von Den

Profefforen Dr. Sans Gebr-Setbelverg, Dr. Selnrid Gerlanb-gena,

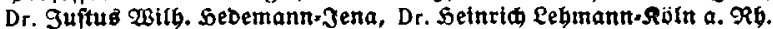

und dem rebaltionellen Ceiter Profeffor Dr. Gris Gter.Gomlo-Söln a. $\mathfrak{R b}$.

\section{Band III}

\section{Gachenrecht}

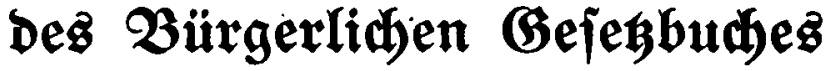

bon

\section{Dr. Iuftuz Bilbelm Sebettanu \\ orbentltgem profeffor ber Redite \\ in Seria}

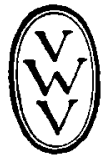

Bexlin unb Peipzig 1924

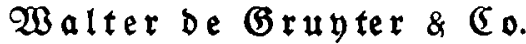

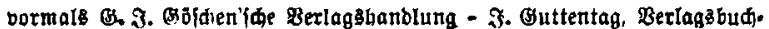

barblung - Beorg Reimer - Farl 3. Trübner - Beit \& Comp. 


\section{$\mathfrak{U} \mathfrak{u g}$ ber Gammlung}

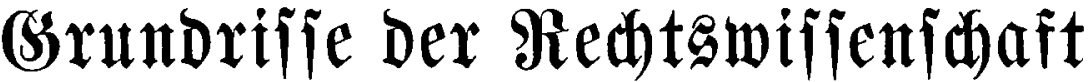

find bizbber erfhienen:

Band I. Allgemeiner Teil bes Bürgerliden Gejegbuches von Oberlandezgeridttzrat Profeffor Dr. Seinrich Lebmann, Röln. 1922. Oftav. 2. 2uflage. Gold $=\mathfrak{N}$. 6. -, geb. 7.50 .

„Dieses Buch ist gleich wertvoll als Ergänzung für die Vorlesung wie als Lernbuch, das das leidige Mitschreiben erspart. Lehmann deckt den Unterbau des gesamten Zivilrechts dem Auge des Lesers auf. Es fehlt nicht an kurzen historischen Perspektiven, und auch die Rechtsentwicklung seit Schaffung des Bürgerlichen Gesetzbuches wird durch fleißige Benutzung der Rechtsprechung des Reichsgerichts dargestellt. Reichliche Beispiele erhöhen die Brauchbarkeit des Buches, dem mant noch manche weitere Auflage voraussagen kann." Hamburger Universitätszeitung.

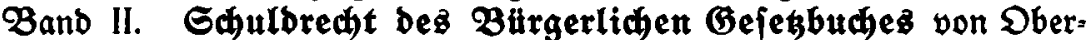
landeżgerichtärat Profeffor Dr. $3 u$ ftu Jena. 1920. Oftab. Gold = M . 7.50, geb. 9. -

... Wo man auch liest, überall weht einem ein frischer Hauch entgegen. Wer Hedemanns Darstellung des Rechts der Schuldverhältnisse nicht mit Interesse und schließlich mit steigender Freude liest, der hat schon garnichts von einem Juristen an sich."

Das Recht.

„Es ist ein Genuß, diese anschauliche, fesselnde, ganz vom Pulsschlag modernen Lebens erfüllte und dabei tiefschürfende Schrift, die überall neue Ausblicke eröffnet, zu lesen."

Staats- und Selbstverwaltung.

Banb III. Gadenred)t von Oberlandeżgerichtżat Profeffor Dr. Suftus Wilbelm Sebemann, Sena. 1924. Oftay.

$\mathfrak{B a l b}=\mathfrak{M}$. ca. 7.50 , geb. ca. 9.-

Band V. (Erbred)t von Geheimrat Profeffor Dr. Friebrid Ende= mann,. Seibelberg. 1923. Ottav. Gold= M. 3.-, geb. 4.50 .

„Der vorliegende Band der Grundrisse der Rechtswissenschaft will wie seineVorgänger vor allem dem Rechtsunterricht dienen, er kann aber auch besonders dem im Unterrichtsdienst stehenden für die praktische Arbeit warm empfohlen werden, und selbst der Praktiker wird in ihm Anregung und Belehrung finden. Der Verfasser versteht es meisterhaft, den gesetzlichen Vorschriften Leben einzuflößen."

Sächs. Archiv f. Rechtspflege.

Band VI. Sanbeläredt und Gaiffahrtßredt von Prof. Dr. Suliuz v. Gierle, Salle a. G. 1921. Oftav. Gold=:M. 7.20, geb. 8.70 .

„Es handelt sich hier um ein praktisch gestaltetes und doch auf wissenschaftlicher Höhe stehendes kleines Lehrbuch, das sich verständigerweise an den Gang des Handelsgesetzbuches anschließt. Die besondere Betonung des lebenden Rechts muß rühmend hervorgehoben werden. Das Büchlein wird dem jungen Studierenden sicherlich gute Dienste leisten." Deutsche Allgemeine Zeitung.

\section{$\mathscr{W} \mathfrak{a} \mathfrak{l} \mathfrak{t} \mathfrak{r} \mathfrak{d} \mathfrak{e} \mathfrak{G} \mathfrak{r} \mathfrak{u} \mathfrak{y} \mathfrak{t} \mathfrak{e} \mathfrak{r} \& \mathbb{C} \mathfrak{o}$. $\mathfrak{B} \in \mathfrak{x} \mathfrak{i n} W .10$ and $\mathfrak{L} \in \mathfrak{i} \mathfrak{j} \mathfrak{i g}$}

Fortiebung autf $\mathfrak{S}$. 3 bes lumidlag? 



\section{Grundriffe \\ ber}

\section{$\mathfrak{R e}(\mathfrak{b}) \mathfrak{z} \mathfrak{w} \mathfrak{i}\{\mathfrak{e} \mathfrak{e} \mathfrak{i} \mathfrak{d}) \mathfrak{a f t}$}

Inter Mitarbelt von

Prof. Dr. Ernft v. Beling-Mäben, Prof. Dr. B. I. Eberb. Röln a. Rh., Dr. Ulerander (Elfter-Berlin, Prof. Dr. Friebrí Enbemann-Sbeibelberg, Prof. Dr. Sanz Febr-Selbelberg, Prof. Dr. Soeinrid Berlano.Sena, Đrof. Dr. Sultus v. Bierte. Salle a.b. G., Prof. Dr. Iuftuछ Wiłth. Sebemann-Iena, Prof. Dr. Serbert Rraus.-Röniggberg i. Pr., Prof. Dr. Seinrid Leh. mann-Roln a. $\mathfrak{R b}$., Prof. Dr. Glaudius Greih. v. GuwerinFretburg i. B., Prof. Dr. Fríg Gtier. Gomlo Röln a. $\mathfrak{R h}$.

berausgegeben won ben

Profefforen Dr. Sang Gehr-Soeibelberg, Dr. Seintic Gerland-Iena, Dr. Sujtug Wilg. Sedemann-Jena,

Dr. Seinrid) Pebmann. Pöln a. $\Re \mathfrak{h}$. unb bem rebattionellen Leiter

Profeffor Dr. Frib Gtier-Gomlo. Pöln a. $\mathscr{R h}$.

\section{$\mathfrak{D r t t e r} \mathfrak{B a n d}$}

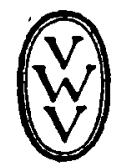

Bertin unb Retpzig 1924

$\mathfrak{W}$ alter be $\mathfrak{G} \mathfrak{r}$ unter \& $\mathfrak{C}_{\text {o. }}$

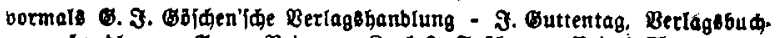
banblung - Seorg Retmer - Rari $\mathfrak{~}$. Trübner - Beit \& Eomp. 


\section{Gadhenredht}

Des

\section{Bürgerlichen (Gejétbuchez}

Bon

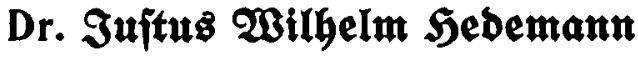

prbentituem profeffor ber Rebte

In Sena

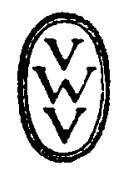

Berlin unb Leipzig 1924

$\mathfrak{W a l t e r}$ be $\operatorname{Gruyter} \&$ Co.

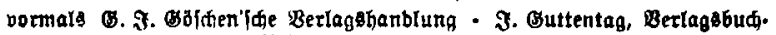
Gandung - Beots Retmet - Sart 3. Trübret - Beit \& Comp. 
Copuright by Walter be (5rubter \& Eo. Berlin und Retpzig 1924. 


\section{$\mathfrak{S} \mathfrak{e} \mathfrak{r} \mathfrak{n}$ \\ Sanz $\mathfrak{A}$ (bredht Giíd \\ Profefior in Sena}

in Freunbichaft zugeeignet 
